Research Paper

\title{
Association of Genetic Polymorphisms in the LncRNAs with Gastric Cancer Risk in a Chinese Population
}

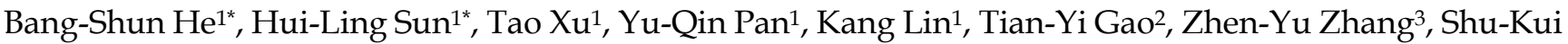 \\ Wang $1 \bowtie$ \\ 1. General Clinical Research Center, Nanjing First Hospital, Nanjing Medical University, Nanjing, China; \\ 2. Department of Laboratory Medicine, Nanjing First Hospital, Nanjing Medical University, Nanjing, China; \\ 3. Department of Gastroenterology, Nanjing First Hospital, Nanjing Medical University, Nanjing, China. \\ *These authors contributed equally to this work. \\ $\square$ Corresponding author: Shu-Kui Wang. Mailing address: General Clinical Research Center, Nanjing First Hospital, Nanjing Medical University, 68 Changle \\ Road, Nanjing 210006, China. E-mail: sk_wang@njmu.edu.cn. \\ (c) Ivyspring International Publisher. This is an open access article distributed under the terms of the Creative Commons Attribution (CC BY-NC) license \\ (https:// creativecommons.org/licenses/by-nc/4.0/). See http://ivyspring.com/terms for full terms and conditions.
}

Received: 2016.09.08; Accepted: 2016.11.10; Published: 2017.02.11

\begin{abstract}
Background: Genome-wide association studies have identified that polymorphisms in 8q24 confer susceptibility to gastric cancer. Polymorphisms in the IncRNA PRNCRI, PCATI, and CCAT2 transcribed from the 8q24 locus have a potential risk for gastric cancer.

Methods: To evaluate whether there is such an association in Chinese population, a case-control study enrolled 494 patients and 494 healthy controls was carried out. Sequenom MassARRAY platform was used for genotyping.

Results: This study showed that $\mathrm{rs} 16901946 \mathrm{G}$ allele was associated with increased risk of gastric cancer (AG: adjusted $\mathrm{OR}=1.33,95 \% \mathrm{Cl}=1.02-1.73, \mathrm{p}=0.033$; GG: adjusted $\mathrm{OR}=2.07 ; 95 \% \mathrm{Cl}=$ 1.11-3.86, $\mathrm{p}=0.023, \mathrm{AG} / \mathrm{GG}$ : adjusted $\mathrm{OR}=1.39,95 \% \mathrm{Cl}=1.08-1.1 .79, \mathrm{p}=0.011$; additive model: adjusted $\mathrm{OR}=1.37 ; 95 \% \mathrm{Cl}=1.10-1.70, \mathrm{p}=0.004)$. Stratified analysis revealed that the increased risk was more evident in the cohort of younger subjects (adjusted $\mathrm{OR}=1.84,95 \% \mathrm{Cl}=1.18-2.87$, $\mathrm{p}=0.007$ ), males (adjusted $\mathrm{OR}=1.55,95 \% \mathrm{Cl}=1.15-2.08, \mathrm{p}=0.004$ ), positive Helicobacter pylori infection (adjusted $\mathrm{OR}=1.44,95 \% \mathrm{Cl}=1.02-2.03, \mathrm{p}=0.041$ ), gastric cardia adenocarcinoma (adjusted $\mathrm{OR}=1.61,95 \% \mathrm{Cl}=1.10-2.35, \mathrm{p}=0.014)$, and tumor stage $\mathrm{T} 1-\mathrm{T} 2$ (adjusted $\mathrm{OR}=1.58$, $95 \% \mathrm{Cl}=1.10-2.28, \mathrm{p}=0.013)$.

Conclusions: Our study suggested that $\mathrm{rs} 16901946 \mathrm{G}$ allele carriers have an increased risk of gastric cancer, and the risk could be enhanced by the interactions between the polymorphism and age, sex, Helicobacter pylori infection.
\end{abstract}

Key words: Gastric cancer; polymorphism; lncRNA.

\section{Introduction}

Gastric cancer is one of the most common cancers worldwide. Despite the incidence of gastric cancer has declined in the past few decades, there is still a high prevalence of gastric cancer in the Eastern Asia including China [1]. Moreover, although the mortality of gastric cancer has a conspicuous decline with the improvement of clinical treatment in the last decade, the prognosis of gastric cancer is still poor with about $20 \%$ five-year survival rates [2]. Gastric cancer is a heterogeneous and complex disease resulting from the interaction of genetic and environmental factors, including helicobacter pylori infection [3], dietary habit [4], smoking and drinking [5].

Long non-coding RNAs (lncRNAs) are non-coding transcripts longer than 200 nucleotides. Recent studies have revealed that the lncRNAs involved in the tumorigenesis for their function as 
proto-oncogene [6] or anti-oncogene [7]. Moreover, the differential expression of lncRNAs was observed in different cancers, including prostate cancer [8], hepatocellular carcinoma [9], colon cancer [10], and gastric cancer [11]. Studies have also suggested that polymorphism in lncRNAs may influence the process of splicing and stability of messenger RNA (mRNA) conformation, leading to the modification of their interacting partners $[12,13]$. Thus, the susceptibility of polymorphisms in lncRNAs to risk of cancers has been investigated among several ethnicities [14, 15]. Moreover, the polymorphisms in the region of 8q24 chromosomal gene-poor region, known as 'gene desert', have been consistently implicated in genome-wide association studies as susceptibility loci for cancers, including prostate cancer, colorectal cancer, and breast cancer $[16,17,18]$.

Prostate cancer non-coding RNA 1(PRNCR1), a lncRNA transcribed from 8q24, involved in the carcinogenesis of prostate cancer through activating androgen receptor (AR) [13], and polymorphisms in the lncRNA PRNCR1 were observed as risk of various cancers, including prostate cancer [13], colorectal cancer[19], and gastric cancer[20]. Moreover, in the 8q24 region, lncRNAs PCAT1 and CCAT2 have potential roles in promoting cancer pathogenesis through affecting double-stranded DNA break repair [21] and chromosome instability [22], and polymorphisms in these lncRNAs were potential risks of cancers [23]. Based on this background, we performed this study to assess the susceptibility of polymorphisms (PRNCR1: rs7463708, rs7007694, rs16901946, rs13252298; PCAT1: rs1026411, rs12543663; CCAT2: rs6983267) in the lncRNAs in the region of 8q24 to risk of gastric cancer, and the prognostic value of these polymorphisms for gastric cancer patients was also evaluated by the overall survival (OS) days.

\section{Materials and Methods}

\section{Study subjects}

A total of 494 gastric cancer patients and same number age- and sex-matched healthy controls were enrolled for this case-control study. All the patients were histologically diagnosed as gastric cancer from January 2009 to January 2014 in Nanjing First Hospital, Nanjing Medical University. Individuals who came to the hospital for routine physical examination were recruited as health controls. All the participants were heritably unrelated ethnic Han Chinese from the same geographic region of Nanjing City, the capital of Jiangsu province, which lies in the middle of the east of China. The characteristics of each subject, including age, gender, smoking, drinking, were collected via a questionnaire. Individuals who had smoked daily for more than one year were considered smokers, and those who consumed one or more alcoholic drinks per week for at least one year were considered drinkers.

For patients, the clinical features, such as tumor size, distant metastasis, depth of invasion, etc., were collected from the patients' medical records. Moreover, the TNM stages were examined and evaluated by the TNM classification according to American Joint Commission for Cancer Staging in 2002, the sixth edition. The patients were followed up at 3-month intervals, and the follow-up information on survival period of patients was updated by a trained clinical specialist through on-site interview, direct calling or medical chart review. The latest follow-up data in this analysis were obtained in June 2016, and finally, a total of 488 patients were traced with periods up to four years,

The protocol of this study was approved by the Institutional Review Board of the Nanjing First Hospital, and written informed consents were obtained from all participants.

\section{DNA extraction and genotyping}

The genotypes were detected as we previously described [24, 25]. In brief, DNA was extracted from whole-blood samples and concentrated by using GoldMag-Mini Whole Blood Genomic DNA Purification Kit according to the manufacture's protocol (GoldMag Co. Ltd. Xi'an, China), and then DNA purity was measured by spectrometry (DU530 UV/VIS spectrophotometer, Beckman Instruments, Fullerton, CA, USA). All samples were genotyped for the seven polymorphisms by the Sequenom MassARRAY platform, according to the standard protocol recommended by the manufacturer with a Sequenom Mass-ARRAY ® RS1000 (Sequenom, Inc.). Multiplexed SNP MassEXTENDED assay was designed by Sequenom MassARRAY Assay Design 3.0 Software [26]. Finally, data management and analysis were performed by Sequenom Typer 4.0 Software [26, 27].

\section{H. pylori serum assays}

The H. pylori infection of all participants were determined by using a commercial $H$. pylori immunogold testing kit (Kangmei Tianhong Biotech (Beijing) Co., Ltd, Beijing, China) according to the suggested procedures. The test has been validated with sensitivity of $98.29 \%$ and specificity of $98.51 \%$ for the detection of $H$. pylori infection in the Chinese populations.

\section{Statistical analysis}

The statistical analysis for genotype distribution 
was performed by the $\chi^{2}$ test with SPSS 11.0 for Windows (SPSS, Chicago, IL, USA). Odds ratios (OR) and $95 \%$ confidence intervals (CIs) were calculated using a logistic regression model. Hardy-Weinberg equilibrium in the control group was tested using a goodness of fit chi-square test. Hazard ratios (HRs) were calculated by multivariate Cox regression analysis. The $\mathrm{P}$ value $<0.05$ was considered statistically significant difference.

\section{Results}

\section{Characteristics of the study population}

The demographic and exposure data of all participants are summarized in Table 1 . There was no significant difference in helicobacter pylori infection rate (cases:56.28\%, controls:51.62\%, $\mathrm{p}=0.110$ ), age $(p=0.718)$, sex $(p=0.943)$, smoking $(p=0.765)$ and drinking status $(p=0.530)$ between cases and controls. For the tumor stage, a total of $130(26.32 \%)$ and 364 $(73.68 \%)$ patients were classified to TNM stage T1-T2 and T3-T4, respectively. For the tumor site, a total of $142(28.74 \%)$ and $352(71.26 \%)$ patients were diagnosed as gastric cardia adenocarcinoma (GCA) and non-gastric cardia adenocarcinoma (NGCA), respectively.

\section{Association between polymorphisms and risk of gastric cancer}

The genotype distributions of the polymorphisms in cases and controls are presented in Table 2. The observed frequencies of all tested genotypes in controls did not deviate from Hardy-Weinberg equilibrium (HWE) (rs16901946: $\mathrm{p}=0.150$; rs13252298: $\mathrm{p}=0.145$; rs7463708: $\mathrm{p}=0.390$; rs7007694: $p=0.111 ; r s 12543663: p=0.802 ; r s 1026411$ : $\mathrm{p}=0.077$; rs6983267: $\mathrm{p}=0.587$ ).

The genotype distributions between the cases and controls were significantly different for rs16901946 ( $\mathrm{p}=0.015)$, but not for $\mathrm{rs} 13252298$ $(\mathrm{p}=0.217), r s 7463708(\mathrm{p}=0.362), r s 7007694 \quad(\mathrm{p}=0.603)$, rs12543663 $(\mathrm{p}=0.904), \quad \mathrm{rs} 1026411 \quad(\mathrm{p}=0.627)$ and rs6983267 $(p=0.870)$. When the rs16901946 AA genotype was used as the reference, the $G$ allele carriers have a higher risk of gastric cancer (AG: adjusted $\mathrm{OR}=1.33,95 \% \mathrm{CI}=1.02-1.73, \mathrm{p}=0.033$; GG: adjusted $\mathrm{OR}=2.07,95 \% \mathrm{CI}=1.11-3.86, \mathrm{p}=0.023$; AG/GG: adjusted OR $=1.39,95 \% \mathrm{CI}=1.08-1.79$, $\mathrm{p}=0.011$ ) after adjustment for age, gender, smoking, drinking, and Helicobacter pylori infection status. In the additive model, rs16901946 was also observed for the increased risk of gastric cancer (adjusted $\mathrm{OR}=1.37$; $95 \% \mathrm{CI}=1.10-1.70, \mathrm{p}=0.004)$. However, there was no significant association between with the other six polymorphisms and gastric cancer risk.

\section{Stratification analysis}

To further assess the risk of rs16901946 to gastric cancer, a stratified analysis was performed by subgroups of age, sex, Helicobacter pylori infection status, tumor stage and tumor site using co-dominant model (AG/GG vs. AA) (Table 3). The increased risk for rs16901946 G allele carriers (AG/GG) was more evident in sub-group of younger subjects (adjusted $\mathrm{OR}=1.84,95 \% \mathrm{CI}=1.18-2.87, \mathrm{p}=0.007)$, males (adjusted OR $=1.55,95 \% \mathrm{CI}=1.15-2.08, \mathrm{p}=0.004$ ), positive Helicobacter pylori infection (adjusted OR = $1.44,95 \% \mathrm{CI}=1.02-2.03, \mathrm{p}=0.041$ ), GCA (adjusted OR $=1.61,95 \% \mathrm{CI}=1.10-2.35, \mathrm{p}=0.014)$, and tumor stage $\mathrm{T} 1-\mathrm{T} 2$ (adjusted $\mathrm{OR}=1.58,95 \% \mathrm{CI}=1.10-2.28$, $\mathrm{p}=0.013$ ), suggesting the risk was enhanced by the potential interactions between rs16901946 and age, sex, Helicobacter pylori infection.

\section{Association between polymorphisms and clinical outcome}

A total of 488 patients were traced for follow-up information on survival period. The association of the polymorphisms with OS of patients was assessed for the predictive value by patients with heterozygous or homozygous genotype compared with those with wild genotype. The result revealed that no polymorphism was associated with OS of patients (Table 4), indicating these polymorphisms have no significantly predicted value for gastric cancer.

Table 1. Frequency distribution of demographic characteristics of gastric cancer cases and cancer-free controls.

\begin{tabular}{llll}
\hline Variables & Cases, $\mathrm{n}(\%)$ & Controls, $\mathrm{n}(\%)$ & $P$ value \\
\hline Age (mean \pm SD) & $64.61 \pm 11.82$ & $64.88 \pm 11.80$ & $0.718^{\mathrm{a}}$ \\
$>60$ & $324(65.59)$ & $327(66.19)$ & $0.840^{\mathrm{b}}$ \\
$\leq 60$ & $170(34.41)$ & $167(33.81)$ & \\
Gender & & & \\
Male & $362(73.28)$ & $362(72.28)$ & $1.000^{\mathrm{b}}$ \\
Female & $132(26.72)$ & $132(26.72)$ & \\
Drinking & & & \\
Yes & $54(10.93)$ & $48(9.72)$ & $0.530^{\mathrm{b}}$ \\
No & $440(89.07)$ & $446(90.28)$ & \\
Smoking & & & \\
Yes & $116(23.48)$ & $120(24.29)$ & $0.765^{\mathrm{b}}$ \\
No & $378(76.52)$ & $374(75.71)$ & \\
Helicobacter pylori & & & \\
infection status & & & \\
Positive & $280(56.28)$ & $255(51.62)$ & $0.110^{\mathrm{b}}$ \\
Negative & $214(43.72)$ & $239(48.38)$ & \\
Clinical stages & & & \\
T1-T2 & $130(26.32)$ & & \\
T3-T4 & $364(73.68)$ & & \\
Tumor site (T1-T4) & & \\
GCA & $142(28.74)$ & \\
NGCA & $352(71.26)$ &
\end{tabular}

GCA, gastric cardia adenocarcinoma; NGCA, non-gastric cardia adenocarcinoma. a Independent $t$-test.

b Two-sided $\chi 2$ test for distributions between cases and controls. Table 2 Logistic regression analysis of association between the polymorphisms and gastric cancer risk. 
Table 2. Logistic regression analysis of association between the polymorphisms and gastric cancer risk.

\begin{tabular}{|c|c|c|c|c|}
\hline Genotypes & Cases, $\mathrm{n}(\%)$ & Controls, $\mathrm{n}(\%)$ & OR $(95 \% \mathrm{CI})^{\mathrm{a}}$ & p-Value \\
\hline \multicolumn{5}{|l|}{ rs16901946 } \\
\hline AA & $261(52.83)$ & $301(60.93)$ & Reference & \\
\hline AG & 203(41.09) & $176(35.63)$ & $1.33(1.02,1.73)$ & 0.033 \\
\hline GG & $30(6.07)$ & $17(3.44)$ & $2.07(1.11,3.86)$ & 0.023 \\
\hline AG/GG & $233(47.17)$ & 193(39.07) & $1.39(1.08,1.79)$ & 0.011 \\
\hline Additive model & & & $1.37(1.10,1.70)$ & 0.004 \\
\hline \multicolumn{5}{|l|}{ rs13252298 } \\
\hline AA & $236(47.77)$ & $209(42.31)$ & Reference & \\
\hline AG & $215(43.52)$ & $235(47.57)$ & $0.81(0.62,1.06)$ & 0.120 \\
\hline GG & $43(8.70)$ & $50(10.12)$ & $0.79(0.50,1.24)$ & 0.303 \\
\hline AG/GG & $258(52.23)$ & $285(57.69)$ & $0.82(0.64,1.05)$ & 0.098 \\
\hline Additive model & & & $0.85(0.70,1.04)$ & 0.113 \\
\hline \multicolumn{5}{|l|}{ rs7463708 } \\
\hline TT & $241(48.79)$ & $228(46.15)$ & Reference & \\
\hline GT & 209(42.31) & 209(42.31) & $0.95(0.73,1.24)$ & 0.690 \\
\hline GG & $44(8.91)$ & $57(11.54)$ & $0.73(0.47,1.12)$ & 0.151 \\
\hline GT/GG & $253(51.21)$ & $266(53.85)$ & $0.90(0.70,1.16)$ & 0.428 \\
\hline Additive model & & & $0.89(0.74,1.08)$ & 0.225 \\
\hline \multicolumn{5}{|l|}{ rs7007694 } \\
\hline TT & $264(53.44)$ & $272(55.06)$ & Reference & \\
\hline CT & $199(40.28)$ & $198(40.08)$ & $1.04(0.80,1.35)$ & 0.760 \\
\hline $\mathrm{CC}$ & $31(6.28)$ & $24(4.86)$ & $1.32(0.75,2.34)$ & 0.339 \\
\hline $\mathrm{CT} / \mathrm{CC}$ & $230(46.56)$ & $222(44.94)$ & $1.07(0.83,1.38)$ & 0.599 \\
\hline Additive model & & & $1.09(0.88,1.34)$ & 0.441 \\
\hline \multicolumn{5}{|l|}{ rs12543663 } \\
\hline $\mathrm{AA}$ & $426(86.23)$ & $425(86.03)$ & Reference & \\
\hline $\mathrm{CA}$ & $66(13.36)$ & $66(13.36)$ & $1.00(0.69,1.44)$ & 0.993 \\
\hline $\mathrm{CC}$ & $2(0.40)$ & $3(0.61)$ & $0.68(0.11,4.17)$ & 0.680 \\
\hline $\mathrm{CA} / \mathrm{CC}$ & $68(13.77)$ & $69(13.97)$ & $0.99(0.69,1.42)$ & 0.945 \\
\hline Additive model & & & $0.98(0.69,1.38)$ & 0.890 \\
\hline \multicolumn{5}{|l|}{ rs1026411 } \\
\hline GG & $186(37.65)$ & 188(38.06) & Reference & \\
\hline GA & $230(46.56)$ & $218(44.13)$ & $1.05(0.79,1.38)$ & 0.753 \\
\hline AA & 78(15.79) & $88(17.81)$ & $0.91(0.63,1.32)$ & 0.616 \\
\hline GA/AA & $308(62.35)$ & $306(61.94)$ & $1.01(0.78,1.31)$ & 0.950 \\
\hline Additive model & & & $0.97(0.81,1.16)$ & 0.725 \\
\hline \multicolumn{5}{|l|}{ rs6983267 } \\
\hline $\mathrm{TT}$ & $167(33.81)$ & $172(34.82)$ & Reference & \\
\hline GT & $233(47.17)$ & $234(47.37)$ & $1.03(0.77,1.36)$ & 0.864 \\
\hline GG & $94(19.03)$ & $88(17.81)$ & $1.08(0.75,1.55)$ & 0.677 \\
\hline GT/GG & $327(66.19)$ & $322(65.18)$ & $1.04(0.80,1.35)$ & 0.776 \\
\hline Additive model & & & $1.04(0.87,1.24)$ & 0.666 \\
\hline
\end{tabular}

aAdjusted for age, gender, smoking, drinking, and Helicobacter pylori infection.

Table 3. Stratification analysis for associations between rs 16901946 and gastric cancer risk

\begin{tabular}{|c|c|c|c|c|c|c|}
\hline \multirow[t]{3}{*}{ Variables } & \multicolumn{4}{|c|}{ Cases/Controls } & \multirow{3}{*}{$\begin{array}{l}\text { AG/GG vs. AA } \\
\text { OR }(95 \% \text { CI })^{a}\end{array}$} & \multirow[t]{3}{*}{ p-Value } \\
\hline & \multicolumn{2}{|c|}{ AA } & \multicolumn{2}{|l|}{ AG/GG } & & \\
\hline & No. & $\%$ & No. & $\%$ & & \\
\hline \multicolumn{7}{|l|}{ Age, year } \\
\hline$\leq 60$ & $86 / 109$ & $50.59 / 65.27$ & $84 / 58$ & $49.41 / 34.73$ & $1.84(1.18,2.87)$ & 0.007 \\
\hline$>60$ & $175 / 192$ & $54.01 / 58.72$ & $149 / 135$ & $45.99 / 41.28$ & $1.22(0.89,1.67)$ & 0.212 \\
\hline \multicolumn{7}{|l|}{ Gender } \\
\hline Male & $189 / 228$ & $52.21 / 62.18$ & $173 / 135$ & $47.79 / 37.19$ & $1.55(1.15,2.08)$ & 0.004 \\
\hline Female & $72 / 73$ & $54.55 / 55.73$ & $60 / 58$ & $45.45 / 44.27$ & $1.02(0.62,1.68)$ & 0.943 \\
\hline \multicolumn{7}{|c|}{$\begin{array}{l}\text { Helicobacter pylori infection } \\
\text { status }\end{array}$} \\
\hline Positive & $146 / 156$ & $52.14 / 61.18$ & $134 / 99$ & $47.86 / 38.82$ & $1.44(1.02,2.03)$ & 0.041 \\
\hline Negative & $115 / 145$ & $53.74 / 60.67$ & $99 / 94$ & $46.26 / 39.33$ & $1.32(0.91,1.93)$ & 0.140 \\
\hline \multicolumn{7}{|l|}{ Tumor site } \\
\hline GCA & $70 / 301$ & $49.30 / 60.93$ & $72 / 193$ & $50.70 / 39.07$ & $1.61(1.10,2.35)$ & 0.014 \\
\hline NGCA & $191 / 301$ & $54.26 / 60.93$ & $161 / 193$ & $45.74 / 39.07$ & $1.32(1.00,1.74)$ & 0.051 \\
\hline \multicolumn{7}{|c|}{ Clinical stages } \\
\hline T1-T2 & $78 / 301$ & $49.37 / 60.93$ & $80 / 193$ & $50.63 / 39.07$ & $1.58(1.10,2.28)$ & 0.013 \\
\hline T3-T4 & $183 / 301$ & $54.46 / 60.93$ & $153 / 193$ & $45.54 / 39.07$ & $1.31(0.99,1.74)$ & 0.057 \\
\hline
\end{tabular}

aAdjusted for age, gender, smoking, drinking, and Helicobacter pylori infection. 
Table 4. Analysis of associations between the polymorphisms and clinical outcome of gastric cancer

\begin{tabular}{lll}
\hline Polymorphism & HR $(95 \% \mathrm{CI})^{\mathrm{a}, \mathrm{b}}$ & $\mathrm{p}$-Value \\
\hline rs16901946 & $0.98(0.78-1.23)$ & 0.850 \\
rs7463708 & $0.90(0.71-1.13)$ & 0.347 \\
rs12543663 & $1.12(0.81-1.55)$ & 0.477 \\
rs13252298 & $1.13(0.90-1.43)$ & 0.293 \\
rs7007694 & $0.97(0.77-1.22)$ & 0.778 \\
rs6983267 & $0.97(0.76-1.23)$ & 0.790 \\
rs1026411 & $0.82(0.65-1.04)$ & 0.108 \\
\hline
\end{tabular}

$\mathrm{HR}$, hazard ratio.

a Cox regression based on heterozygous or homozygous genotype compared with wild genotype.

bAdjusted for age, gender, smoking, drinking, and Helicobacter pylori infection.

\section{Discussion}

This population-based case-control study recruited 494 gastric cancer patients and 494 age- and sex- matched healthy controls in a Chinese population, and demonstrated that rs16901946 was associated with increased risk of gastric cancer, especially for individuals with younger age $(\leq 60)$, male, positive Helicobacter pylori infection status, GCA, and gastric cancer in stage T1-T2.

The lncRNA PRNCR1 was reported to promote the carcinogenesis of prostate through activating androgen receptor [13], which is a member of the nuclear receptor family acting as a transcription factor [28]. Rs16901946 in the lncRNA PRNCR1 was reported to be associated with risk of cancers [23], and it might affect the predicted secondary structure of PRNCR1 mRNA, and change the stability of lncRNA PRNCR1 or the mRNA conformation leading to the modification of its interacting partners [13]. Previously, $\mathrm{Li}$ et al. reported that there was no association of rs16901946 to the risk of colorectal cancer [19] and gastric cancer [20], which was not consistent to the result of this study. The inconsistent conclusion may due to several factors; firstly, their studies were performed on the population from Sichuan province, west of China, but this study based on the population from Jiangsu province, east of China; secondly, this study observed the significant association of rs16901946 to gastric cancer risk was more obvious in the subgroup of GCA(adjusted $\mathrm{OR}=1.61,95 \% \mathrm{CI}=1.10-2.35, \mathrm{p}=0.014)$ and then in NGCA(adjusted OR=1.32, 95\% CI=1.00-1.74, $\mathrm{p}=0.051$ ); however, regrettably, the cases in their study were not classified; thirdly, this study suggested that the interaction between Helicobacter pylori infection and rs16901946 leads to higher risk of gastric cancer, and the ratio of Helicobacter pylori infection in this study was $56.28 \%$ and $51.62 \%$ in cases and controls, respectively, but this data was not included in their studies, and finally, the sample size of this study was larger than theirs, which may also cause the different conclusions.

Sub-group analysis revealed that rs16901946 G allele carriers and with younger age $(\leq 60)$, male, and positive Helicobacter pylori infection status have higher gastric cancer risk, indicating the interaction of Helicobacter pylori infection and rs16901946 enhanced the risk of gastric cancer, which was consistent to the fact that Helicobacter pylori infection is a risk of gastric cancer. In China, actually, there is a fact that the ration of drinking and smoking is higher in male than in female; moreover, the prevalence of gastric cancer in male is higher than in female, which may contribute the different risk among the gender. Individuals with younger age have different life style compared with the older, such as work pressure, less time to relaxing, etc, which may enhance the risk of gastric cancer. The sub-group analysis revealed that rs16901946 was associated with the cancer type of GCA, and in stage T1-T2, indicating lncRNA PRNCR1 contribution to the carcinogenesis of gastric cancer may modify by the cancer type. To our knowledge, there was no study reporting association between rs16901946 and gastric cancer risk. Thus, study with a large sample size and stratified analysis should be performed to confirm this result.

The value of all enrolled polymorphisms for predicting the survival of patients with gastric cancer was also analyzed, and no significant association was observed between these polymorphisms and OS, indicating these polymorphisms including rs16901946 could not be applied as a marker to predict the progress of gastric cancer. Similarly, in the prostate cancer, study concluded that lncRNA PRNCR1 was not a prognostic marker [29], which was consistent to the result of this study.

Some limitations of this study should be noted. Firstly, the sample size of this study was relatively small, which may limit the statistical power, particularly in the multiple stratified analyses. Secondly, several potential environmental factors, such as diet, physical exercise, history of gastric diseases, were not included in this study, which may influence gastric cancer risk. Finally, the polymorphisms were included based on previous knowledge of their potential functional roles in the occurrence of cancers, but their functions were not clear.

In conclusion, this case-control study provided the evidence that rs16901946 in the lncPRNCR1 were associated with gastric cancer risk, and the risk could be enhanced by the interactions between the polymorphism and age, sex, Helicobacter pylori infection. 


\section{Abbreviations}

lncRNA, long non-coding RNA; mRNA, messenger RNA; PRNCR1, prostate cancer non-coding RNA 1; AR, androgen receptor; OS, overall survival; $\mathrm{HR}$, hazard ratio; $\mathrm{CI}$, confidence interval; GCA, gastric cardia adenocarcinoma (GCA); NGCA, non-gastric cardia adenocarcinoma.

\section{Acknowledgements}

This study was supported by grants from the National Nature Science Foundation of China (No. 81472027, 81501820) to SKW and YQP; Nanjing Medical Science and technique Development Foundation to BS. H (no. JQX13003, QRX11254, QYK11175) and YQ. P (no. QRX11255), and Nanjing Medical University Science and Technique Development Foundation Project to HL. S (no. 2015NJMUZD049).

\section{Ethics Committee Approval and Patient Consent}

The protocol of this study was approved by the Institutional Review Board of the Nanjing First Hospital, and written informed consents were obtained from all participants.

\section{Authors' contributions}

$\mathrm{BSH}$ and SKW conceived and designed the experiments. BSH, HLS, TX, YQP, KL, TYG and ZYZ performed the experiments. BSH and HLS analyzed the data. TX, YQP, KL, TYG and ZYZ contributed reagents/materials/analysis tools. BSH and HLS wrote the manuscript. All authors read and approved the final manuscript.

\section{Competing Interests}

The authors have declared that no competing interest exists.

\section{References}

1. Torre LA, Bray F, Siegel RL, et al. Global cancer statistics, 2012. CA Cancer J Clin. 2015; 65(2):87-108.

2. Nagini S. Carcinoma of the stomach: A review of epidemiology, pathogenesis, molecular genetics and chemoprevention. World journal of gastrointestinal oncology. 2012; 4(7):156-9.

3. Gonzalez CA, Sala N, Rokkas T. Gastric cancer: epidemiologic aspects. Helicobacter. 2013; 18 Suppl 1:34-8.

4. Jakszyn P, Gonzalez CA. Nitrosamine and related food intake and gastric and oesophageal cancer risk: a systematic review of the epidemiological evidence. World J Gastroenterol. 2006; 12(27):4296-303.

5. Sjodahl K, Lu Y, Nilsen TI, et al. Smoking and alcohol drinking in relation to risk of gastric cancer: a population-based, prospective cohort study. Int J Cancer. 2007; 120(1):128-32.

6. Li L, Feng T, Lian Y, et al. Role of human noncoding RNAs in the control of tumorigenesis. Proceedings of the National Academy of Sciences of the United States of America. 2009; 106(31):12956-61.

7. Zhang X, Rice K, Wang Y, et al. Maternally expressed gene 3 (MEG3) noncoding ribonucleic acid: isoform structure, expression, and functions. Endocrinology. 2010; 151(3):939-47.

8. Fu X, Ravindranath L, Tran N, et al. Regulation of apoptosis by a prostate-specific and prostate cancer-associated noncoding gene, PCGEM1. DNA and cell biology. 2006; 25(3):135-41.
9. Lin R, Maeda S, Liu C, et al. A large noncoding RNA is a marker for murine hepatocellular carcinomas and a spectrum of human carcinomas. Oncogene. 2007; 26(6):851-8.

10. Deng $\mathrm{Q}, \mathrm{He} \mathrm{B}, \mathrm{Gao} \mathrm{T}$, et al. Up-regulation of $91 \mathrm{H}$ promotes tumor metastasis and predicts poor prognosis for patients with colorectal cancer. PloS one. 2014; 9(7):e103022.

11. Fan ZY, Liu W, Yan C, et al. Identification of a five-lncRNA signature for the diagnosis and prognosis of gastric cancer. Tumour biology : the journal of the International Society for Oncodevelopmental Biology and Medicine. 2016. DOI: $10.1007 /$ s13277-016-5185-9.

12. Burd CE, Jeck WR, Liu Y, et al. Expression of linear and novel circular forms of an INK4/ARF-associated non-coding RNA correlates with atherosclerosis risk. PLoS genetics. 2010; 6(12):e1001233.

13. Chung $S$, Nakagawa $H$, Uemura $M$, et al. Association of a novel long non-coding RNA in 8q24 with prostate cancer susceptibility. Cancer science. 2011; 102(1):245-52.

14. Han Y, Rand KA, Hazelett DJ, et al. Prostate Cancer Susceptibility in Men of African Ancestry at 8q24. Journal of the National Cancer Institute. 2016; 108(7).

15. Xue $Y, \mathrm{Gu} D, \mathrm{Ma}$ G, et al. Genetic variants in lncRNA HOTAIR are associated with risk of colorectal cancer. Mutagenesis. 2015; 30(2):303-10.

16. Ghoussaini M, Song $\mathrm{H}$, Koessler T, et al. Multiple loci with different cancer specificities within the $8 \mathrm{q} 24$ gene desert. Journal of the National Cancer Institute. 2008; 100(13):962-6.

17. Turnbull C, Ahmed S, Morrison J, et al. Genome-wide association study identifies five new breast cancer susceptibility loci. Nature genetics. 2010; 42(6):504-7.

18. Tomlinson I, Webb E, Carvajal-Carmona L, et al. A genome-wide association scan of tag SNPs identifies a susceptibility variant for colorectal cancer at 8q24.21. Nature genetics. 2007; 39(8):984-8

19. Li L, Sun R, Liang $\mathrm{Y}$, et al. Association between polymorphisms in long non-coding RNA PRNCR1 in 8q24 and risk of colorectal cancer. Journal of experimental \& clinical cancer research : CR. 2013; 32:104.

20. Li L, Jia F, Bai P, et al. Association between polymorphisms in long non-coding RNA PRNCR1 in 8q24 and risk of gastric cancer. Tumour biology : the journal of the International Society for Oncodevelopmental Biology and Medicine. 2016; 37(1):299-303.

21. Prensner JR, Chen $\mathrm{W}$, Iyer MK, et al. PCAT-1, a long noncoding RNA, regulates BRCA2 and controls homologous recombination in cancer. Cancer research. 2014 74(6):1651-60.

22. Ling H, Spizzo R, Atlasi $Y$, et al. CCAT2, a novel noncoding RNA mapping to $8 \mathrm{q} 24$, underlies metastatic progression and chromosomal instability in colon cancer. Genome research. 2013; 23(9):1446-61.

23. Ling $\mathrm{H}$, Vincent $\mathrm{K}$, Pichler $\mathrm{M}$, et al. Junk DNA and the long non-coding RNA twist in cancer genetics. Oncogene. 2015; 34(39):5003-11.

24. Lou $G$, Song $X$, Yang $F$, et al. Exosomes derived from miR-122-modified adipose tissue-derived MSCs increase chemosensitivity of hepatocellular carcinoma. J Hematol Oncol. 2015; 8(1):122.

25. He BS, Pan YQ, Lin $K$, et al. Evaluation the susceptibility of five polymorphisms in microRNA-binding sites to female breast cancer risk in Chinese population. Gene. 2015; 573(1):160-5.

26. Gabriel S, Ziaugra L, Tabbaa D. SNP genotyping using the Sequenom MassARRAY iPLEX platform. Curr Protoc Hum Genet. 2009, Chapter 2:Unit 2 12 .

27. Thomas RK, Baker AC, Debiasi RM, et al. High-throughput oncogene mutation profiling in human cancer. Nat Genet. 2007; 39(3):347-51.

28. Heemers HV, Tindall DJ. Androgen receptor (AR) coregulators: a diversity of functions converging on and regulating the AR transcriptional complex. Endocr Rev. 2007; 28(7):778-808.

29. Prensner JR, Sahu A, Iyer MK, et al. The IncRNAs PCGEM1 and PRNCR1 are not implicated in castration resistant prostate cancer. Oncotarget. 2014;5(6):1434-8. 